\title{
2-D multiline spectroscopy of the solar photosphere ${ }^{\star}$
}

\author{
F. Berrilli ${ }^{1}$, G. Consolini ${ }^{2}$, E. Pietropaolo ${ }^{3}$, B. Caccin ${ }^{1}$, V. Penza ${ }^{1}$, and F. Lepreti ${ }^{4}$ \\ 1 Dipartimento di Fisica, Università di Roma Tor Vergata, V.le Ricerca Scientifica 1, 00133 Roma, Italy \\ 2 Istituto di Fisica dello Spazio Interplanetario-CNR, Area di Ricerca Tor Vergata, 00133 Roma, Italy \\ 3 Dipartimento di Fisica, Università di L'Aquila, Località Coppito, 67010 L'Aquila, Italy \\ 4 Dipartimento di Fisica, Università della Calabria, 87036 Rende (CS), Italy
}

Received 11 May 2001 / Accepted 4 September 2001

\begin{abstract}
The structure and dynamics of the photosphere are investigated, with time series of broadband and monochromatic images of quiet granulation, at the solar disk center. Images were acquired with the IPM observing mode at the THEMIS telescope. Velocity and line center intensity fields, derived from the observation of three different photospheric lines, are used to study velocity and intensity patterns at different heights in the photosphere. Automatic segmentation procedures are applied to velocity and intensity frames to extract solar features, and to investigate the dependence of their properties at different scales and heights. We find a dependence of the statistical properties of upflow and downflow regions on the atmospheric height. Larger granules, passing through a great part of the photosphere, are used to investigate the damping of convective motions in stably stratified layers. The results suggest the occurrence of an intense braking in the deep photosphere (first $\sim 120 \mathrm{~km}$ ). Furthermore, we investigate the temporal and spatial evolution of velocity fields, deriving typical time scales of dynamical processes relative to different solar features. In particular, for two selected isolated exploders, we reveal a velocity deceleration in the central region since the early phase of their fragmentation.
\end{abstract}

Key words. Sun: atmosphere - Sun: photosphere - Sun: granulation

\section{Introduction}

At present photospheric granulation patterns, visible on the solar surface, can be studied using both high spectral and high spatial resolution imaging. The characterization of velocity and intensity features, forming the photospheric pattern, is of great importance in a variety of scientific fields, as pointed out by Spruit et al. (1990). A dynamical description of the atmospheric region where convective motions brake, and its association with a geometrical description of the observed structures, has profound implications for a variety of topics ranging from energy transport in astrophysical plasmas to physics of fluids with high Reynolds numbers. Moreover, because solar convection is the only example of astrophysical convection that can be directly investigated, it represents the main test bench for stellar modeling.

Nowadays, hydrodynamic simulations of stellar-solar convection have reached a high level of refinement

Send offprint requests to: F. Berrilli,

e-mail: berrilli@roma2.infn.it

* Based on observations made with THEMIS-CNRS/INSUCNR operated on the island of Tenerife by THEMIS S.L. in the Spanish Observatorio del Teide of the Instituto de Astrofisica de Canarias.
(Steffen et al. 1989; Kim et al. 1996; Stein \& Nordlund 1998; Gadun et al. 1999; Gadun et al. 2000). Nevertheless, the insufficient knowledge of convection processes still remains one of the most important uncertainties in stellar astrophysics (Gustafsson \& Jorgensen 1994; Kim et al. 1996). A physical description of photospheric layers immediately above the convectively unstable solar envelope is even more difficult. Here the warm mass elements, which gained momentum in the lower region, overshoot owing to their inertia and brake on account of viscous forces. Moreover, the upper layer of photospheric fluid may be unstable (e.g. Rayleigh-Taylor instabilities might be present) and this dynamical state complicates the physical description. Therefore, only a complete knowledge of gradients, fluxes and dynamics in this boundary layer could allow an actual description of energy exchanges and fluid movements.

Convection effects have been invoked to generate acoustical waves (see Strous et al. 2000); the temporal evolution of recognized structures and the oscillation features of the observed fields are useful tools to analyze the interaction between convective fluxes and acoustic wave generation (Rast 1999; Strous et al. 2000). In the last decade, the possibility that individual source events may excite $p$-modes has been suggested by different authors. 
For example, Rimmele et al. (1995) suggested that oscillations are fed by isolated seismic events occurring in the dark intergranular lanes, and Rast $(1995,1999)$ made numerical simulations of the acoustic response to localized cooling events (plumes) originating at the center of exploding granules. Therefore, it is evident that a detailed description of the dynamics of downflows around convective blobs, as well as of exploding granules, is necessary to investigate such a physical process.

In this work we investigate different aspects of the dynamics of photospheric layers using THEMIS observations (URL: http://www.themis.iac.es). Particularly, we study the stratification of intensity and velocity fluctuations in the regions marked by granules and intergranular lanes, or by upflows and downflows. We also investigate the dynamical properties of selected photospheric structures such as exploding granules.

\section{Observations}

Different time series of broadband ( $4 \mathrm{~nm} F W H M$ around $557 \mathrm{~nm})$ and monochromatic (2.1 pm $F W H M)$ images were acquired at the THEMIS telescope (Observatorio del Teide, Tenerife) in 1999 summer. Specifically, we analyzed the series obtained on July 1st (from 7:21 to 8:24 UT) with the IPM observing mode. This mode consists of a Universal Birefringent Filter followed by a Fabry-Pérot interferometer (Cavallini 1998). A square portion $\left(34^{\prime \prime} \times 34^{\prime \prime}\right)$ of the quiet disk center was imaged with the broadband camera and, simultaneously, with the monochromatic camera at different spectral points along the profile of C I $538.03 \mathrm{~nm}(\Delta \lambda=0, \pm 4, \pm 6 \mathrm{pm}$, continuum), Fe I $537.9 \mathrm{~nm}(\Delta \lambda=0, \pm 4, \pm 6 \mathrm{pm})$, and Fe I $557.61 \mathrm{~nm}$ $(\Delta \lambda=0, \pm 6, \pm 8, \pm 10 \mathrm{pm}$, continuum) lines. Each spectral series consisted of 19 broadband and 19 monochromatic images. The images were acquired with two different integration times, $40 \mathrm{~ms}$ and $200 \mathrm{~ms}$ for broadband and monochromatic frames, respectively. The two 12-bit CCD cameras (Berrilli et al. 1997) are binned to $256 \times 256$. The final pixel size for the binned images was $0.134^{\prime \prime}$. Seeing conditions and telescope set-up limited the spatial resolution to $0.3-0.5^{\prime \prime}$ (approx. $300 \mathrm{~km}$ on the Sun) for broadband images. The time interval between two successive images and two successive spectral series was $2.5 \mathrm{~s}$ and $75 \mathrm{~s}$, respectively.

\section{Data analysis and image processing}

\subsection{Data reduction}

After having corrected the images for dark current, flat field, image motion, and tracking loss of the telescope, a sub-array of approx. $30^{\prime \prime} \times 30^{\prime \prime}$ made up the final image series. The finally selected 40 -min sequence, 32 spectral series, consists of 608 broadband and 608 monochromatic frames. The broadband image contrast $C$ is shown in Fig. 1. In order to reduce the noise and take into account the instrumental profile, we applied a Wiener filter (Hunt 1984) to broadband images. We assumed a

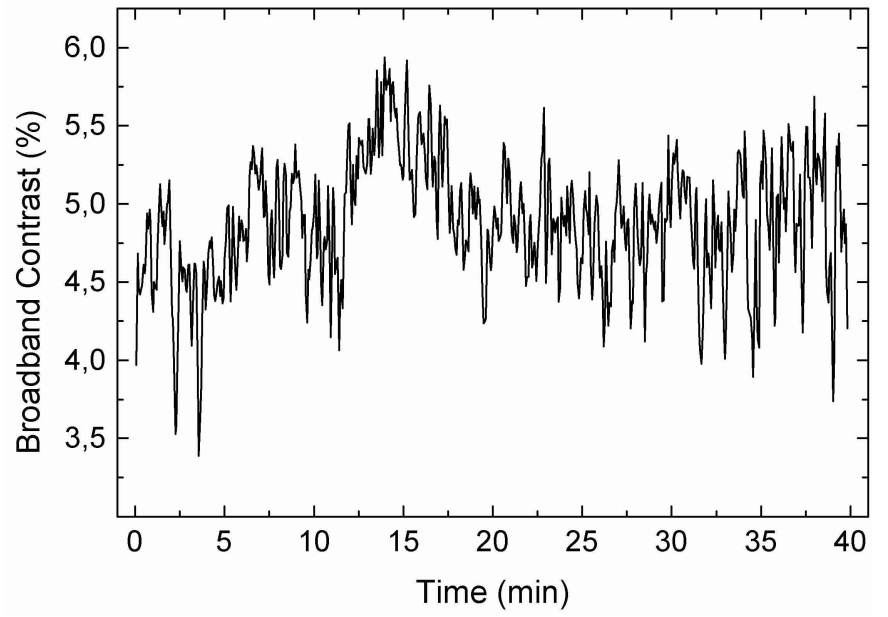

Fig. 1. Contrast of the original broadband image timeseries $(557.6 \mathrm{~nm}, F W H M=5 \mathrm{~nm})$. Images were acquired without frame selection, with an integration time of $40 \mathrm{~ms}$. The average observed contrast is $4.9 \%$; after Wiener restoring we found an average contrast of $7.1 \%$.

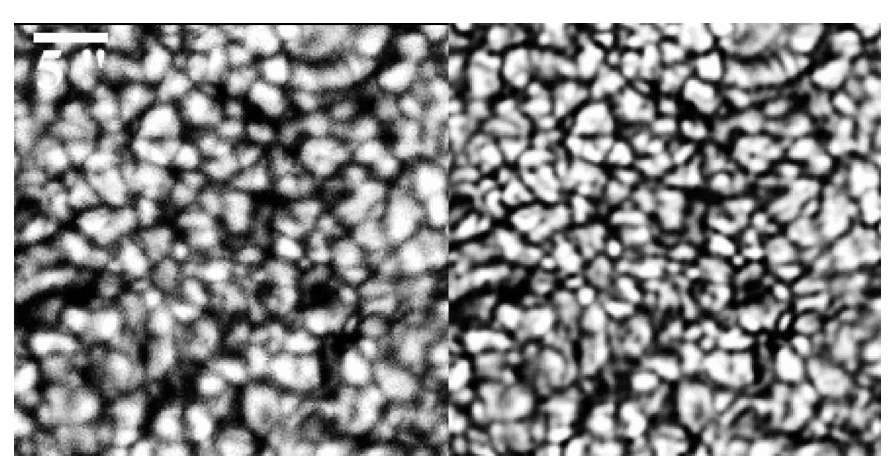

Fig. 2. Original and Wiener restored $\left(F W H M=0.4^{\prime \prime}\right.$, $S / N=15)$ images. The box size is $30^{\prime \prime} \times 30^{\prime \prime}$. The original image, with contrast equal to $4.9 \%$, was obtained by THEMISIPM at 07:50 UT with an integration time of $40 \mathrm{~ms}$ at $557.6 \mathrm{~nm}$ and with a passband of $4 \mathrm{~nm}$.

simple Gaussian PSF $\left(F W H M=0.4^{\prime \prime}\right.$, comparable to spatial resolution of the observations) and a $S / N$ ratio equal to 15 , which was found to represent a good compromise between the level of restoration and the noise amplification. In Fig. 2 we show a typical image $(C=4.9 \%)$ before and after the application of the Wiener filter. The restored images have been used to study the granulation properties in the broadband continuum; conversely, to compare continuum images with velocity fields, we used unrestored images. In fact, the need to use different monochromatic images to obtain a single map of velocity and line-center intensity ultimately reduces the spatial resolution.

Monochromatic images corresponding to a single line profile were acquired sequentially in the IPM observing mode, i.e. one wavelength position at a time; therefore, to derive physical quantities like the Doppler velocity, we used for each spectral series a Fourier interpolation to shift the observations in time to the same instant. 


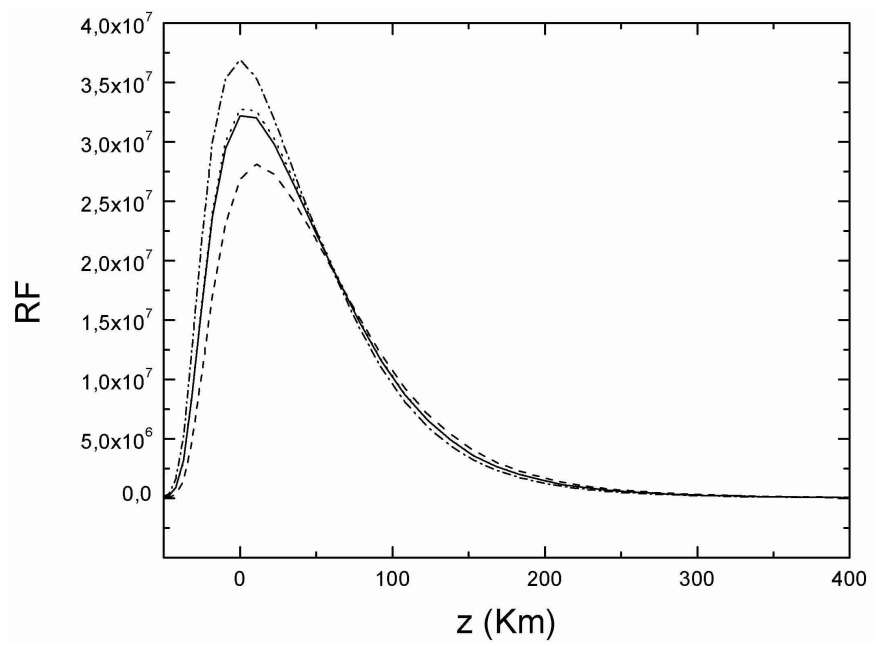

Fig. 3. Velocity response function for the C I line. Solid line: average $R F_{V}$; dashed line: center line position $\lambda_{0}$; dotted line: $\lambda=\lambda_{0}+4 \mathrm{pm}$; dashed-dotted line: $\lambda=\lambda_{0}+6 \mathrm{pm}$.

\subsection{Velocity response function}

To obtain information about the depth dependence of the photospheric velocity fields by associating to any line a suitable "formation zone", we can treat their effects on the line profiles as linear perturbations and study the velocity response functions $R F_{V}$ of the emergent intensity at the observed wavelengths within the lines (Caccin et al. 1977). From them we can form a velocity weighting function of the line bisector, at each wavelength and for each line, dividing $R F_{V}$ by the wavelength derivative of the unpertubed profile (Canfield 1976; Buonaura \& Caccin 1982; Nesis \& Mattig 1989):

$\delta I=\int_{-\infty}^{+\infty} V(z) R F_{V}(\lambda, z) \mathrm{d} z$

where, with usual notations,

$R F_{V}(\lambda, z)=\frac{\partial \chi(\lambda, z)}{\partial V}(S(z)-I(\lambda, z)) \mathrm{e}^{-\tau(\lambda, z)}$.

Here we assumed LTE, so that the source function is velocity independent. Notice however that there is some evidence of NLTE effects for the Fe I $\lambda 557.61$ line (Shchukina et al. 1997); this might cause a slight overestimation of the line formation heights in our calculations, especially in the line core. The calculations were made with the Harvard-Smithsonian Reference Atmosphere (HSRA; Gingerich et al. 1971). To mimic NLTE effects in the core of the Fe I $\lambda 557.6$ line, we used a monotonically decreasing extrapolation of the HSRA photospheric temperature above $h=550 \mathrm{~km}$, similar to that of the HOLMUL model (Holweger \& Müller 1974).

Notice that, as shown in Figs. 3-5, the formation zone is sensibly different at different wavelengths for both Fe I lines, while it is always practically coincident with that of the continuum for the C I line. Because of the fitting procedure used in this paper to derive a single wavelength shift for each line, it will be sufficient to consider, in

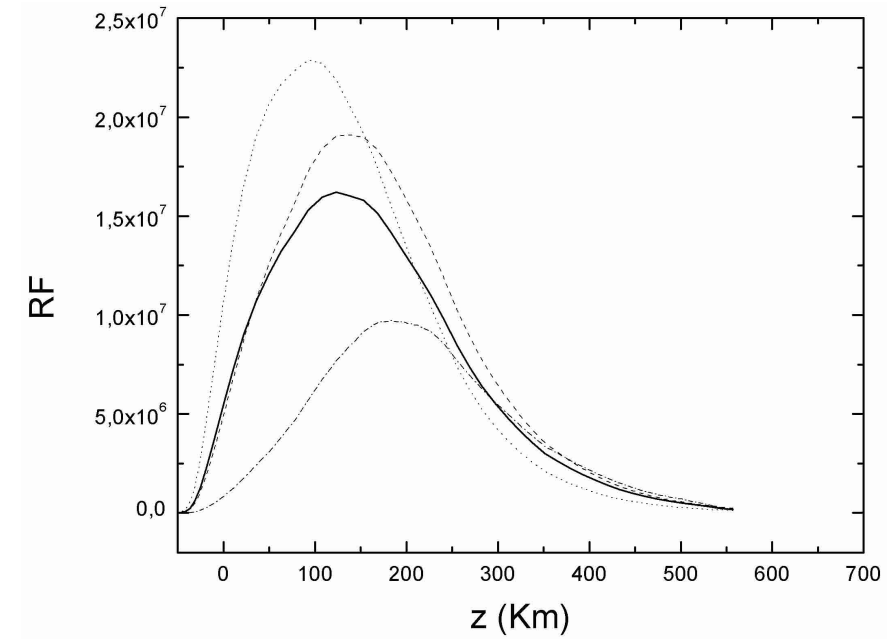

Fig. 4. Velocity response function for the Fe 5379 line. Solid line: average $R F_{V}$; dashed-dotted line: center line position $\lambda_{0}$; dashed line: $\lambda=\lambda_{0}+4 \mathrm{pm}$; dotted line: $\lambda=\lambda_{0}+6 \mathrm{pm}$.

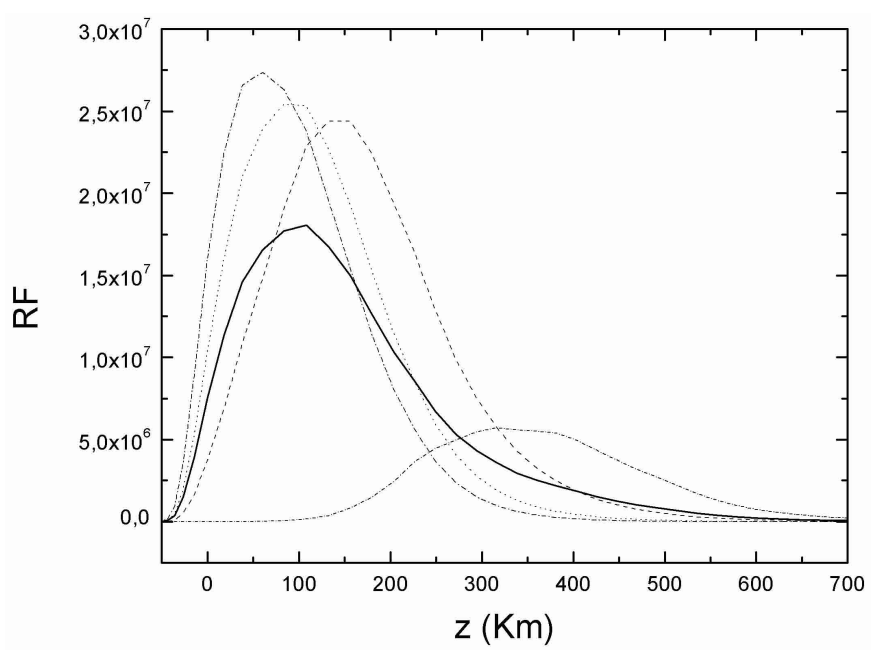

Fig. 5. Velocity response function for the Fe 5576 line. Solid line: average $R F_{V}$; dashed-double dotted line: center line position $\lambda_{0}$; dashed line: $\lambda=\lambda_{0}+6 \mathrm{pm}$; dotted line: $\lambda=\lambda_{0}+8 \mathrm{pm}$; dashed-dotted line: $\lambda=\lambda_{0}+10 \mathrm{pm}$.

Table 1. Line formation depth (in $\mathrm{km}$ ) for the line center and for the whole line. The $F W H M$ of the average $R F_{V}$ is also given (in $\mathrm{km}$ ).

\begin{tabular}{lccc}
\hline Line & $h_{\lambda_{0}}$ & $\langle h\rangle$ & $F W H M$ \\
\hline C I 538.02 nm & 10 & 0 & 100 \\
Fe I 537.96 nm & 180 & 120 & 250 \\
Fe I 557.61 nm & 320 & 110 & 270 \\
\hline
\end{tabular}

any case, the mean of the weighting functions. In Table 1 we give the position of the peak and the full width at half maximum for the mean curves, although the large width and the strong asymmetry of the weighting functions make these parameters rather poor descriptors. For comparison with the computations of Komm et al. (1989) we give also the peak position for the core $\left(\lambda_{0}\right)$ of each line. 


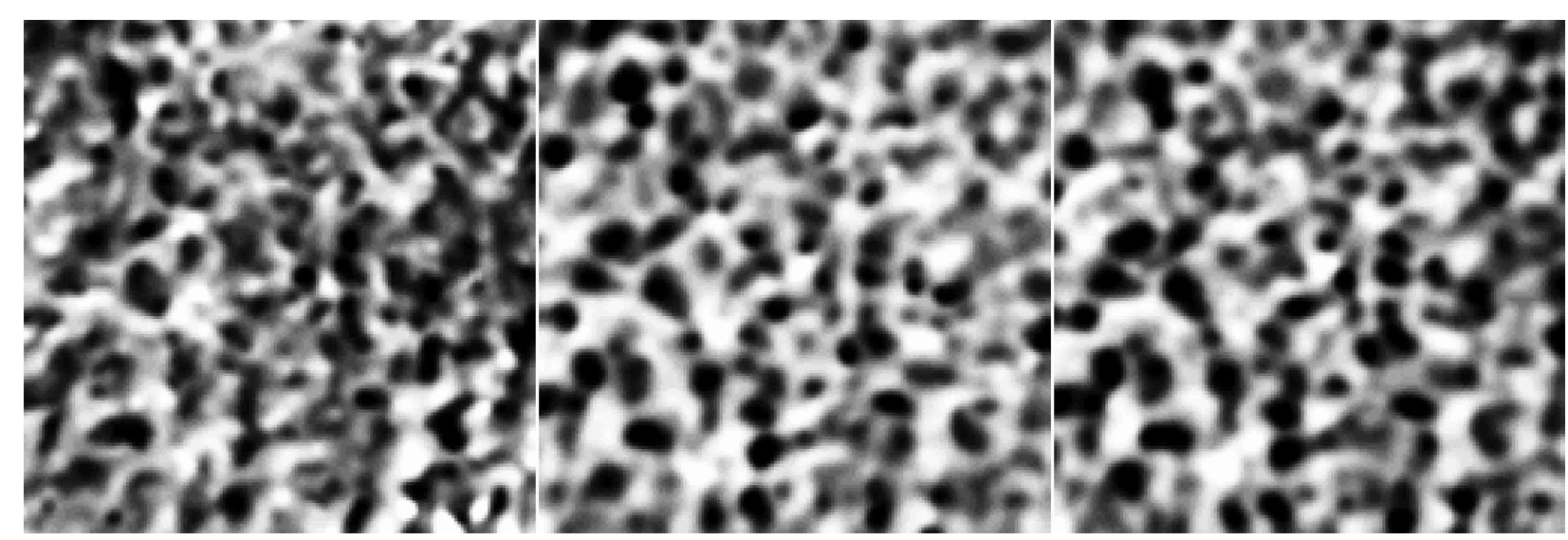

Fig. 6. Velocity maps for the 13th spectral series in the lines C I $538.0 \mathrm{~nm}$ (left), Fe I $537.9 \mathrm{~nm}$ (middle), and Fe I $557.6 \mathrm{~nm}$ (right). These gray scales range approx. from $-1500 \mathrm{~m} \mathrm{~s}^{-1}$ (dark, upflow) to $+1700 \mathrm{~m} \mathrm{~s}^{-1}$ (bright, downflow) for C I and from $-900 \mathrm{~m} \mathrm{~s}^{-1}$ to $+700 \mathrm{~m} \mathrm{~s}^{-1}$ for Fe I lines. The box size is $27^{\prime \prime} \times 27^{\prime \prime}$.

\subsection{Velocity maps}

To compute velocity maps by monochromatic images, we fitted sampled line-profiles with a Gaussian and transformed Doppler shifts in velocity units. The monochromatic images were not restored with the Wiener filter, which we applied only to broadband images. Actually, the longer integration times needed, and the way in which they are combined to get a single velocity map, ultimately reduce spatial resolution. The latter can be estimated by comparing the distribution function of spatial features, extracted from velocity maps, with similar distribution functions derived from artificially degraded broadband images. We found a final spatial resolution lying between $0.6^{\prime \prime}$ and $0.9^{\prime \prime}$ for the velocity maps.

The convergence capability of numerical fit procedures depends on the characteristics of monochromatic observations. Specifically, we found a different percentage of pixels where the fit procedure converges for the three lines. The percentages are $95 \%, 97 \%$, and $100 \%$ for C I 538.0, Fe I 537.9, and Fe I 557.6 lines, respectively. The pixels where the fit procedure fails are set to the median value of a neighboring $4 \times 4$ box.

In Fig. 6 we show three typical velocity maps relative to the observed photospheric lines.

The photosphere dynamics, strongly influenced by acoustic oscillations, must be studied by taking into account the velocity field associated with the 5 -min acoustic pattern. Because the aim of our work is to study convective flows by means of velocity and intensity maps, we have to remove acoustic patterns before analyzing granulation fields (Title et al. 1989). In this regard we applied a $k-\omega$ subsonic filter with a cut-off velocity of $6 \mathrm{~km} \mathrm{~s}^{-1}$ on the time series of broadband, velocity, and centerline intensity maps. After the application of the $k-\omega$ subsonic filter, the effective field-of-view was reduced to approx. $27^{\prime \prime} \times 27^{\prime \prime}$. In the following we conventionally will assume as positive the downward line-of-sight velocities.

In our case the rms velocities are $\sim 400 \mathrm{~m} \mathrm{~s}^{-1}$, $\sim 190 \mathrm{~m} \mathrm{~s}^{-1}$, and $\sim 180 \mathrm{~m} \mathrm{~s}^{-1}$ for C I 538.0 , Fe I 537.9 , and Fe I 557.6 lines, respectively. These rms velocities are consistent with Nesis et al. (1999) findings, but are a factor of 3 lower than the value $575 \mathrm{~m} \mathrm{~s}^{-1}$ found by Krieg et al. (2000) at the height of approx. $100 \mathrm{~km}$, corresponding roughly to our Fe I data. The disagreement could be due to many different causes, such as the different image restoring process, velocity computational procedures and $p$-mode filtering techniques, and to the use of different spectral lines. Furthermore, we do not find evidence of any increase of rms velocity in the first layers of the photosphere, unlike the results of Eibe et al. (2001) obtained by analyzing a dataset of thirteen "typical" granules observed in the $\mathrm{Na} \mathrm{I} \mathrm{D}_{2}$ line.

\subsection{Image segmentation}

Solar features have been identified with a numerical procedure (Florio \& Berrilli 1997; Berrilli et al. 1999a) consisting of two different algorithms.

The first algorithm, based on an iterative procedure of Medial Axis Transform (MAT), better known as skeleton, considers gray-level information at the pixels along the selected regions to extract reticular structures. The MAT of a region of interest $\mathrm{R}$ could be defined in the following way: $\forall p(x, y) \in \mathrm{R}$, we find its closest neighbor belonging to the boundary $\mathrm{B}$; if $p(x, y)$ has more than one such neighbor, then $p(x, y) \in$ MAT. In our case reticular intensity structures represent intergranular lanes, while reticular velocity structures represent downflow lanes. The identified reticular structures are used as the boundary for granular cells and convective cells. To extract such structures the iterative MAT procedure starts from a two-level representation of the original image and, after successive iterations that consider "intensity" information of the image to be skeletonized, stretches the reticular structure in order to fit the valley topology of the image.

The second algorithm, based on a dynamical threshold, extracts compact structures. From intensity fields the procedure extracts granules, while from velocity fields it extracts upward regions (upflows). The dynamical threshold 


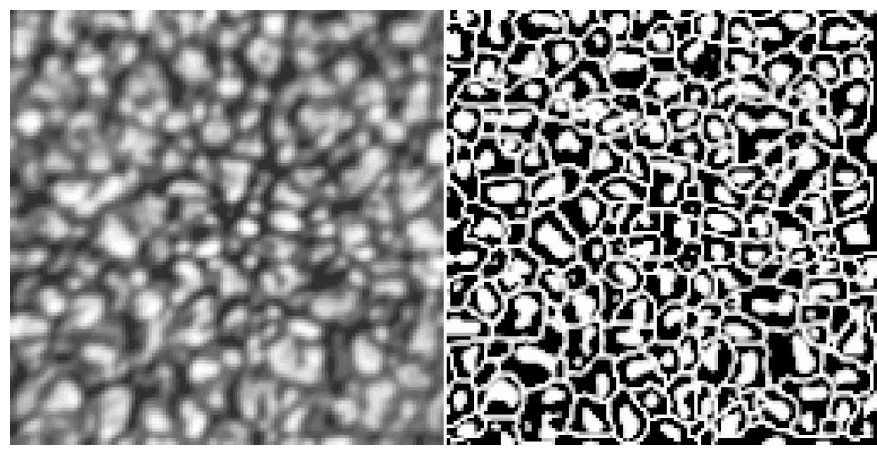

Fig. 7. Left panel: broadband image of a granulation field. Right panel: the network obtained by the application of iterative MAT algorithm, defining the convective cell boundaries, and the compact pixel aggregations obtained by the application of dynamical threshold, defining the granules. The box size is $27^{\prime \prime} \times 27^{\prime \prime}$.

value depends on the pixel position and on the statistical properties of a moving window centered on the selected pixel. Particularly, we used for the granules a $3^{\prime \prime} \times 3^{\prime \prime}$ box and an intensity threshold of $\left\langle I_{\text {window }}\right\rangle+\xi \times \mathrm{rms}_{\text {window }}$, where $<I_{\text {window }}>$ and $\mathrm{rms}_{\text {window }}$ denote, respectively, the average and the rms fluctuations of the intensity in the moving window, and $\xi=0.85$. These choices represent a good compromise to identify small sized granules just fragmented from an exploder. If we reduce the $\xi$ value too much, we lose the multiple structure of exploders, producing big granules, while if we increase $\xi$ too much the algorithm produces numerous small sized granules that are rejected as features that are too small.

A successive segmentation algorithm, based on region growing by pixel aggregation, calculates statistical and geometrical information from identified structures. A typical intensity image and the corresponding photospheric features extracted in this way are shown in Fig. 7.

\section{Results}

The solar convection does not present the characteristics of a stable Bénard convection (Faber 1997); indeed it shows fuzzy structures and an erratic dependence in time. For this complicated system, it is important to identify statistical parameters that can be used as general descriptors of the phenomenon, involving constraints on granulation numerical simulations.

Because our observations strongly indicate that bigger granules pass through a large part of the photosphere we will use such structures to probe the stable stratified layers where convective motions overshoot.

\subsection{Photospheric feature sizes}

Photospheric features can be investigated using both broadband images, where we observe granules and intergranular lanes, and velocity maps, where we find upflows and downflow lanes.
For broadband images, to reduce the effect of a multiple identification of solar structures, we study the properties of granules and cells as extracted from a subset of 608 original images. Specifically, we selected, for each of 32 spectral series, only one image: that with the highest rms intensity fluctuation in the broadband $557.6 \mathrm{~nm}$ region. We used the rms parameter as a raw descriptor of seeing conditions, hence of image quality, assuming negligible intensity variations over the solar surface during the acquisition of the spectral series. After having identified the intensity structures, we excluded features lying on the frame boundary or smaller than $0.3^{\prime \prime} \times 0.3^{\prime \prime}$ (Berrilli et al. $1999 \mathrm{~b})$ from the analysis. At the end of the segmentation procedure, we extracted 4317 granules and 4510 cells. The disparity between the number of granules and cells results both from the diverse selection criteria defining the two kind of structures, and from the absence of a perfect one to one correspondence between extracted granule and containing cell. For each extracted feature, we calculated the area, in pixels, and transformed the area in $\mathrm{km}^{2}$. The effective "size" of the feature is therefore obtained as the diameter of the circle with the same area. Histograms of granules and of cells areas are shown in Fig. 8.

The area distributions can be affected by the time evolution of observed features. Particularly, the presence of long-lived structures could produce an overestimate of large area structure numbers. Actually, lifetime increases as granule size increases (Title et al. 1989). Regarding this point we are now investigating the problem of granule evolution using automatic feature tracking programs, and we plan to present our results in a future paper. Small differences between our distributions and those presented in the literature could derive from the use of different segmentation algorithms; in particular, the small sized features can suffer from segmentation procedures. As discussed in Sect. 3.4. the adopted threshold definition is set to preserve the multiplicity of sub-granules in exploders.

The obtained granule area distribution, which is quite flat for effective sizes less than $1 \mathrm{Mm}$, presents a steep drop at a scale of approx. $1 \mathrm{Mm}$. Instead, the cell distribution presents an elbow at a scale of about $1.5 \mathrm{Mm}$ and a maximum effective size of about $3 \mathrm{Mm}$. The area values corresponding to observed elbows for granular cells and granules seem to indicate a characteristic ratio between cell and granule area of 2.2 , i.e. a cell area which is $55 \%$ of the granule area.

Hitherto, we analyzed the photosphere using only segmented broadband intensity images. Nevertheless segmentation procedures, applied to velocity maps, permit an alternative way to identify and analyze photospheric structures. In particular, using upward blobs as upflows and downward velocity loops as the boundary of convective cells, we repeated the analysis on segmented velocity images. As already discussed, we recognized upflows using a dynamical threshold, and convective cells extracting their boundary by means of a MAT.

Our dataset consists of 32 velocity maps for each spectral line. This corresponds to a total of 96 velocity maps. 


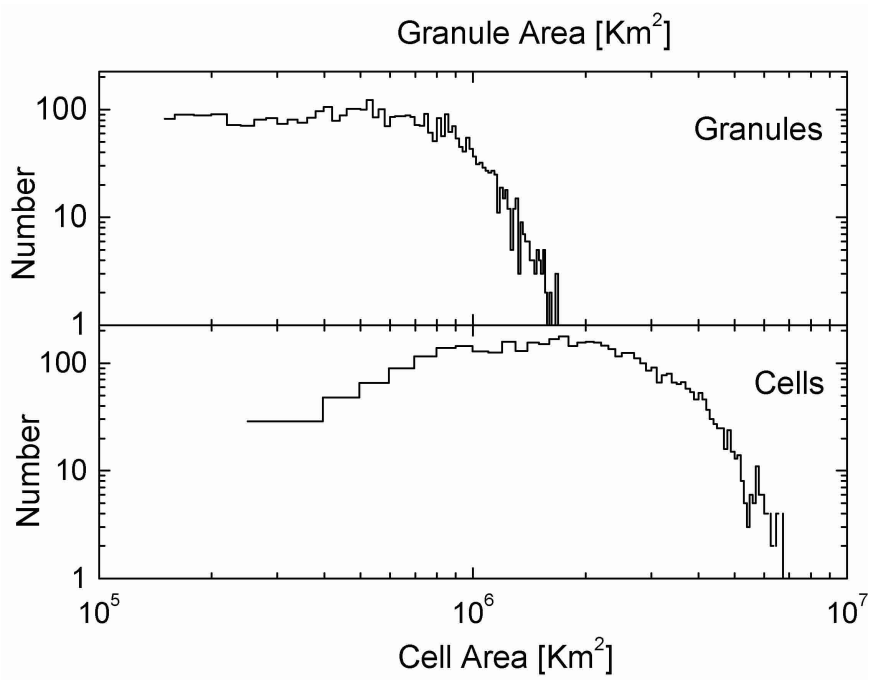

Fig. 8. Area histograms of 4317 granules (upper panel) and 4510 cells (lower panel). The features are extracted from 32 (75 s interval) broadband images. We find a steep drop for the granule area distribution (effective size $\sim 1 \mathrm{Mm}$ ). The elbow for the cells is at $\sim 1.5 \mathrm{Mm}$.

After having applied the segmentation algorithm, we evaluated the distribution functions for identified structures in the velocity maps relative to the three investigated photospheric lines (Figs. 9 and 10). Specifically, using velocity maps derived from $\mathrm{C}$ I line observations, we obtained a distribution function very similar to that evaluated from broadband images. This behavior confirms the high correlation between velocity features near the solar surface and continuum intensity structures.

All distribution functions show a sharp change in the slope, but their exact shape depends on the selected line. In detail, we found a dependence of the distribution on the photospheric height, clearly indicating that the number of small features decreases moving upward in the solar atmosphere. Moreover, the distribution functions derived from the two Fe I lines look very similar despite the different formation height of the line cores; this is a consequence of the similarity of the average $R F_{V}$ for the two lines. Although the likelihood distribution functions for larger granules seems to suggest that they retain their horizontal dimension over the analyzed range of heights, this supposition cannot be proved only on the basis of this statistical approach. Actually in order to prove this statement we need to distinguish and identify the different structures in the different velocity maps. We plan to use the same approach as the feature tracking method previously discussed to validate this possibility.

\subsection{Intensity of granules}

The segmentation and identification algorithms applied to restored broadband images allowed us to calculate average properties of selected granules. In Fig. 11 we plot the average intensity contrast $\left\langle I_{\mathrm{g}}\right\rangle /<I_{\text {frame }}>$ of extracted granules as a function of their effective size. $\left\langle I_{\mathrm{g}}\right\rangle$ and

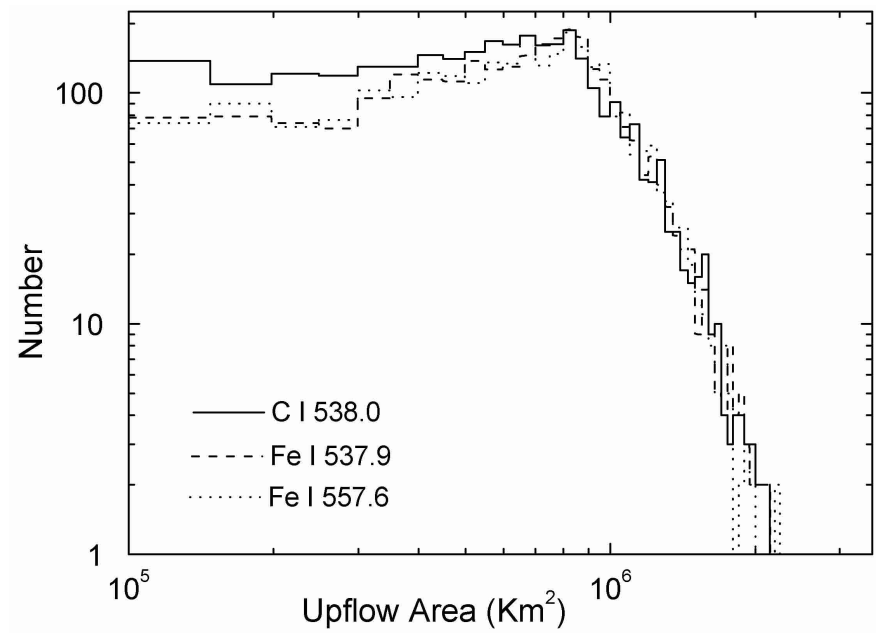

Fig. 9. Area histograms of upflows. The features are extracted from 96 velocity images, 32 for each line. Solid lines, C I $538.0 \mathrm{~nm}$ velocity map, dotted lines, Fe I $537.9 \mathrm{~nm}$ velocity map, and dashed lines, Fe I $557.6 \mathrm{~nm}$ velocity map.

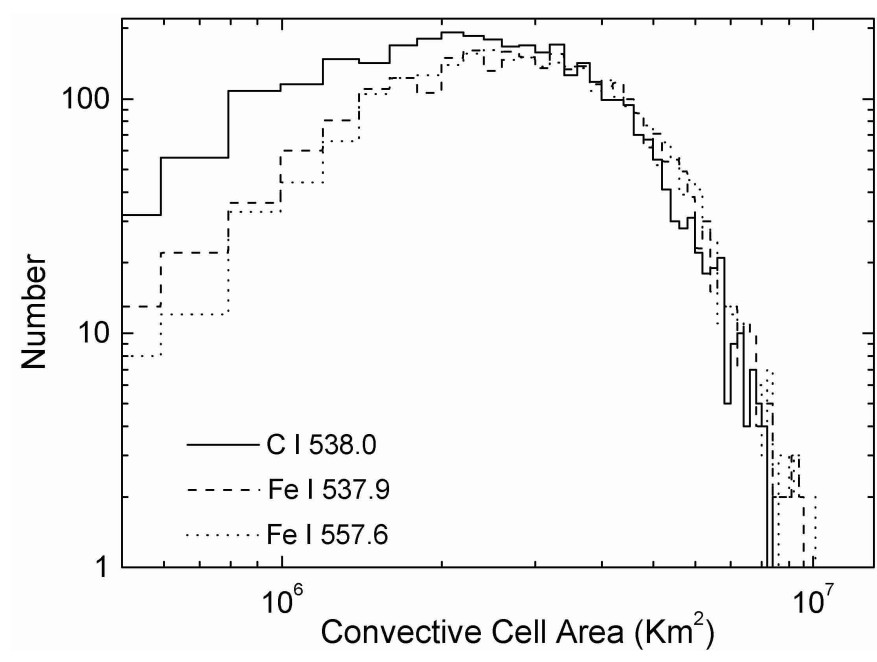

Fig. 10. Area histograms of convective cells (downflow boundaries). The features are extracted from 96 velocity images, 32 for each line. Solid lines, C I $538.0 \mathrm{~nm}$ velocity map, dotted lines, Fe I $537.9 \mathrm{~nm}$ velocity map, and dashed lines, Fe I $557.6 \mathrm{~nm}$ velocity map.

$<I_{\text {frame }}>$ denote the average intensity of the granule and of the whole quiet sun frame, respectively. The figure shows a roughly linear dependence of the contrast on the granular size up to approx. $1 \mathrm{Mm}$ (i.e. $1.4^{\prime \prime}$ ) and a nearly unvarying contrast for larger granules, in agreement with the observations of Hirzberger et al. (1997) and the prediction of non-stationary convection by Gadun et al. (2000). This behavior suggests the possible existence of two granulation regimes, corresponding to small and large granular sizes. The position of the elbow, in our granule area distribution, presents a value smaller than that $\left(\sim 2^{\prime \prime}\right)$ reported by Hirzberger et al. (1997). The numerical discrepancy probably depends on the application of different restoring and segmentation algorithms. On the other hand, our value is nearly in agreement with $2-\mathrm{D}$ numerical 


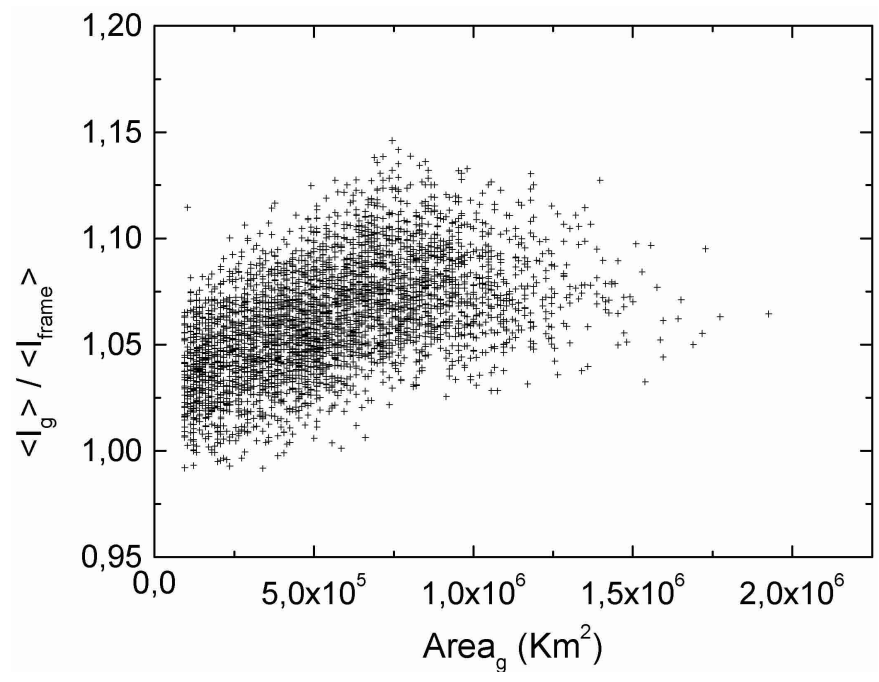

Fig. 11. Average intensity contrast of identified granules vs. granule area. The dependence of mean intensity contrast with the area exhibits a linear behavior for small effective size granules, it becomes almost size-independent at larger areas.

simulations by Gadun et al. (2000); however we note that this accord might be coincidental.

The short lifetime of granules, and the thermal adjustment time of convective structures, could concur to explain the observed behavior. As a matter of fact, under the simple assumption of a spherical convective structure radiating into the surroundings, we can calculate the time scale for thermal adjustment under photospheric conditions. This is roughly the time necessary to release the excess thermal energy, and it is given by (Kippenhahn \& Weigert 1994):

$\tau_{\text {adj }}=\frac{\kappa \rho^{2} c_{\mathrm{p}} S_{\mathrm{g}}^{2}}{16 \sigma c T^{3}}$

where $\kappa=7 \times 10^{-4} \mathrm{~cm}^{2} \mathrm{~g}^{-1}$ is the mean absorption coefficient, $\rho=3 \times 10^{-7} \mathrm{~g} \mathrm{~cm}^{-3}$ is the matter density, $c_{\mathrm{p}}=1.563 \times 10^{8} \mathrm{erg}^{-1}$ is the specific heat at constant pressure, $S_{\mathrm{g}}$ is the diameter, expressed in $\mathrm{cm}$, of the convective blob (i.e. the granular effective size), $\sigma=7.57 \times 10^{-15} \mathrm{erg} \mathrm{cm}^{-3} \mathrm{~K}^{-4}$ is the radiation-density constant, $c=2.9979 \times 10^{10} \mathrm{~cm} \mathrm{~s}^{-1}$ is the speed of light, and $T=6390 \mathrm{~K}$ is the mean temperature of the blob. All physical quantities refer to photospheric conditions. For a typical scale $S_{\mathrm{g}}=1 \mathrm{Mm}$ the thermal adjustment time $\tau_{\text {adj }}$ is of few minutes. Such a small value could mean that, while small granules reach, due to radiative exchanges, an equilibrium temperature with the surroundings before their death, larger ones have a nearly adiabatic evolution. Therefore, the temperature in the larger granules remains stable as a function of depth, retaining a memory of the temperature from the deeper layers. A similar explanation has been proposed by Steffen et al. (1989) and by Gadun et al. (2000) to interpret their numerical results.

\subsection{Vertical extent of the convective flow}

To study the line-of-sight velocity of rising convective elements, and specifically to investigate the braking of upward flows in the overshooting region, we calculated mean velocities of upflows by laying velocity maps on segmented velocity images. The segmented images contain identified pixel aggregations that emerge in a downflow network. The C I line allowed us to estimate the velocities of the features closer to the solar surface, while Fe I lines do the same in the upper photospheric layers. The spatial effective size of velocity structures is calculated, as we did for granules, from their area. In Figs. 12 and 13 we report the average velocities, and the fit of maximum velocities, as functions of derived areas of upward velocity structures for C I 538.0 and Fe I 557.6 lines. In both cases we observe a dependence of average and maximum velocity on the dimension of rising structures; the upflow velocity increases with the dimension of the blob. The results obtained with the Fe I $537.9 \mathrm{~nm}$ line, being quite similar to those observed for the other Fe I line, are not shown here.

When compared to the numerical simulation by Gadun et al. (2000), our results display the same trend of vertical granular velocities vs. size shown by unstable models (fragmenting cells). The quantitative differences of our observations with the Gadun et al. (2000) models might arise from image segmentation procedures, and from spatial smoothing due to atmospheric seeing and finite telescope resolution.

Assuming an exponential dependence for observed distributions, we can calculate the asymptotic value of the upward velocities $\left(V_{\mathrm{a}}\right)$. The three observed distributions present different final asymptotic velocities $V_{\mathrm{a}}$ and different velocity spreads.

The analysis of the area distribution of the observed velocity structures, together with the velocity dependence on the upflow area, suggests two characteristics of velocity patterns:

1. in the first layers of the photosphere there is a selection effect of convective structures depending on their dimension. Large convective structures penetrate higher into the upper photosphere, while the convection in the small structures is limited to the lower layers;

2. average velocities of upward blobs show a dependence on their area. In particular, mean velocities tend to reach an asymptotic velocity $V_{\mathrm{a}}$ when the dimension grows.

Therefore, using larger velocity structures to probe the dynamics of this region, we investigated the braking of upward motions. In Fig. 14 we report the exponential fits of upflow mean velocities $\left\langle V_{\mathrm{up}}\right\rangle$, for the three lines, as a function of the area. From the decrease of asymptotic velocities we infer that upflow braking is very efficient in the first photospheric layers, $\Delta V \sim 300 \mathrm{~m} \mathrm{~s}^{-1}$ in the first $\sim 120 \mathrm{~km}$.

If we limit our analysis to the downflow network, it is possible to study the features of downward velocity 


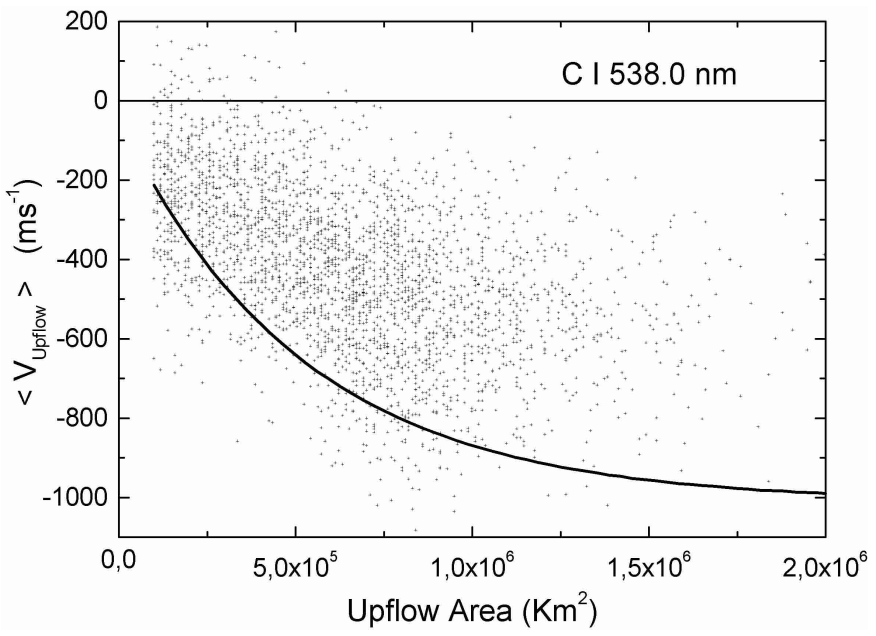

Fig. 12. Dependence of average velocity (points) on area of identified upflow structure. Velocities are calculated using C I $538.0 \mathrm{~nm}$ frames. The solid line represents an exponential decay fit of maximum upflow velocities.

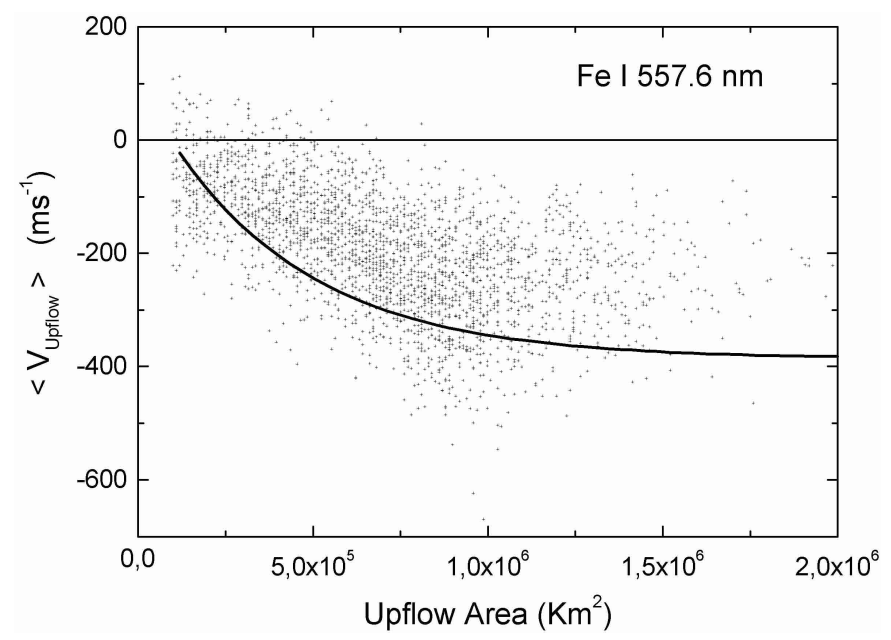

Fig. 13. Dependence of average velocity (points) on area of the identified upflow structure. Velocities are calculated using Fe I $557.6 \mathrm{~nm}$ frames. The solid line represents an exponential decay fit of maximum upflow velocities.

distribution. We restricted our attention to the investigation of the velocity pattern evaluated with the $\mathrm{C}$ I line, which, being located near the solar surface, permits a direct investigation of velocity features associated with granulation. Figure 15 shows the histogram of vertical velocity, at the base of the photosphere, obtained from the pixels indicated by the MAT of downflows. When reported on a semi-logarithmic plot, the distribution of the downflow vertical velocity is in close agreement with a nearly simple exponential law, $\exp -\beta v$ with $\beta \sim-2$, over the range $\left(0.5-1.5 \mathrm{~km} \mathrm{~s}^{-1}\right)$. The non-Gaussian shape of the downflow vertical velocity distribution may be read as evidence of out-of-equilibrium dynamics perhaps related to the occurrence of turbulence in the downflow regions. As a matter of fact, it is well known that large deviations from Gaussian statistics are observed in the case of turbulence,

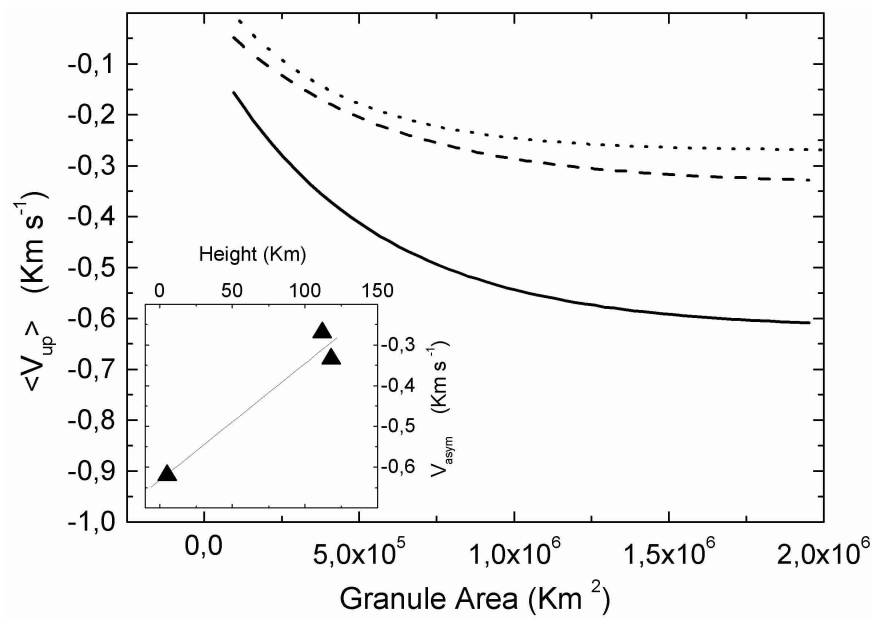

Fig. 14. Average velocity variation fits of the extracted upflow features as derived from observations of the three different lines: C I 538.0 (full line), Fe I 537.9 (dashed line), and Fe I 557.6 (dotted line). From adopted exponential fits we calculate the asymptotic velocity $\left(V_{\mathrm{a}}\right)$. In the small box height dependence of asymptotic velocity is shown; triangles represent calculated asymptotic velocities for the assigned heights, line represents the fit on points.

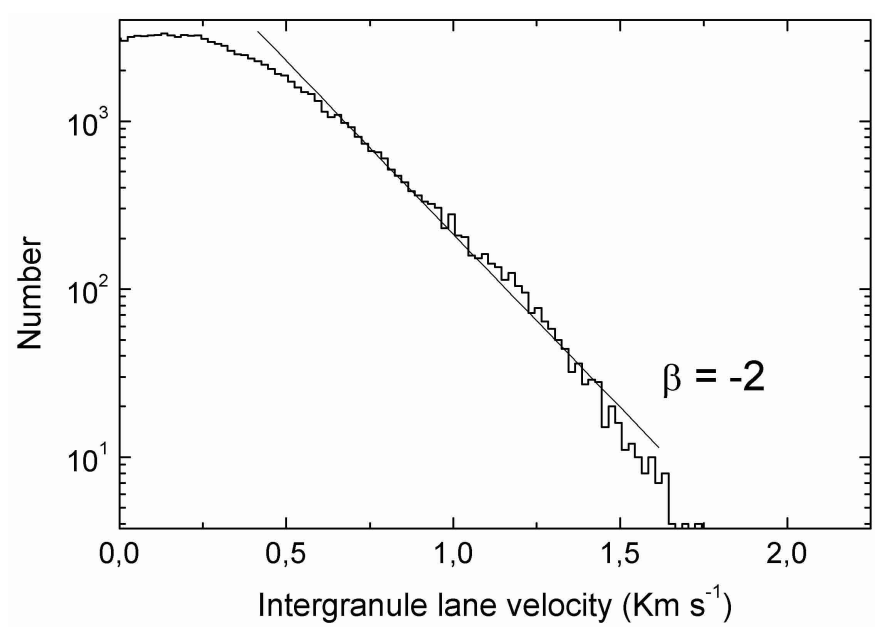

Fig. 15. Histogram of vertical velocity, along intergranular lanes, at the base of the photosphere. Solid line represents a linear fit in the range $0.5-1.5 \mathrm{~km} \mathrm{~s}^{-1}$.

especially in presence of intermittence. This aspect will be investigated in a future work.

\subsection{Velocity and line-center intensity}

By looking at the line-center intensity and average velocity of extracted features in the different lines, we can derive information about the temperature structures in the first photospheric layers. Consider Fig. 16, which shows the linear fits of average line-center intensity $\left\langle I_{\text {up }}>\right.$ vs. average velocity $\left\langle V_{\text {up }}>\right.$ of upflow features for the three lines. At the bottom of the photosphere $\left\langle I_{\text {up }}>\right.$ increases with $\left\langle V_{\text {up }}\right\rangle$, in fact the fluid inside the identified structure is warmer and we observe the well-known correlation 


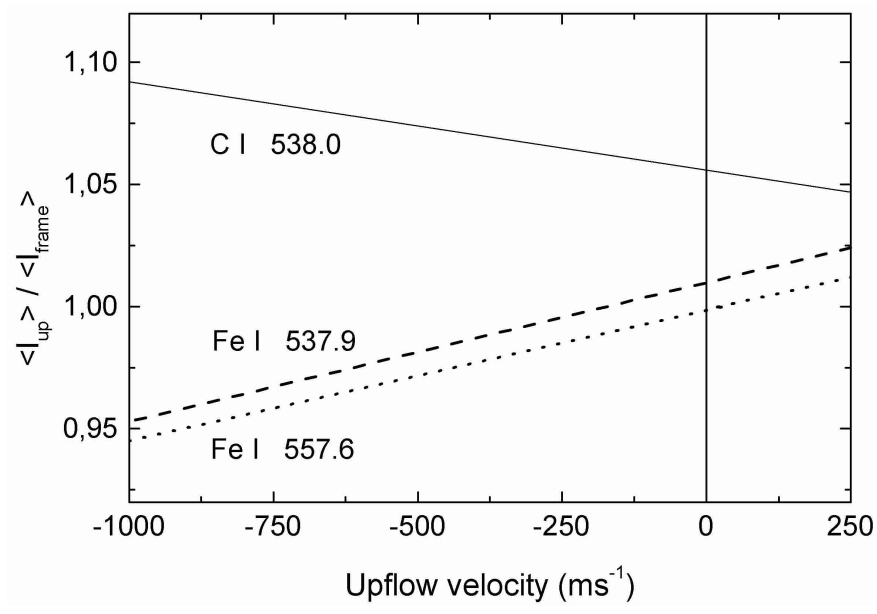

Fig. 16. Linear fits of average line-center intensity $\left\langle I_{\text {up }}\right\rangle$ versus average velocity $\left\langle V_{\text {up }}>\right.$ of "upflow" features. Solid line, is derived from the C I $538.0 \mathrm{~nm}$ velocity map, dotted line, from Fe I $537.9 \mathrm{~nm}$ velocity map, and dashed line, from Fe I $557.6 \mathrm{~nm}$.

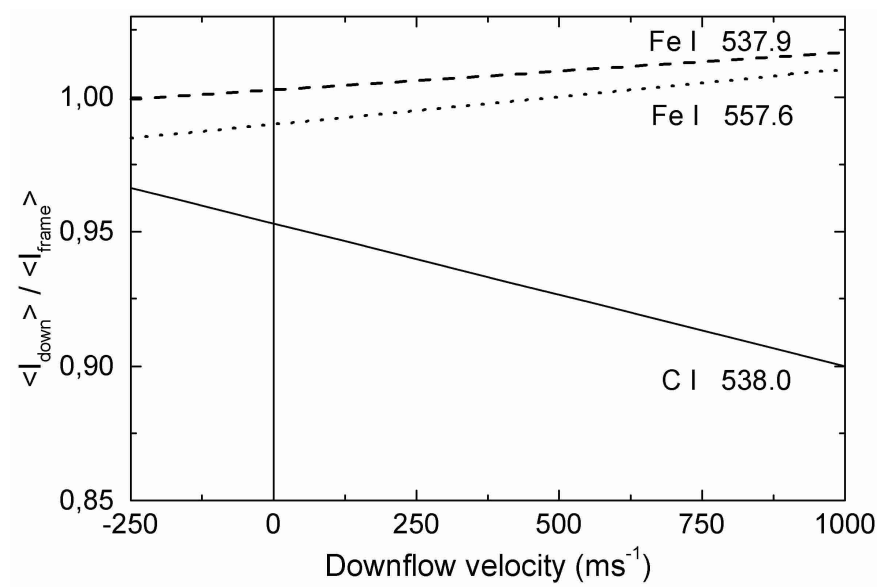

Fig. 17. Linear fits of average line-center intensity $\left\langle I_{\text {down }}>\right.$ vs. average velocity $<V_{\text {down }}>$ of "downflow" features. Solid lines, C I $538.0 \mathrm{~nm}$ velocity map, dotted lines, Fe I $537.9 \mathrm{~nm}$ velocity map, and dashed lines, Fe I $557.6 \mathrm{~nm}$.

between granules and velocity pattern. Moving upward in the atmosphere, the plasma inside the granule, adiabatically cooled, has a lower temperature with respect to the surrounding, so that $\left\langle I_{\text {up }}\right\rangle$ decreases and $\left\langle V_{\text {up }}\right\rangle$ increases. The inversion of temperature fluctuations, in this atmospheric layer, is explained in term of warm flow penetration into stably stratified layers (Steffen et al. 1989); in practice these layers are relatively cooler above upward moving blobs. Figure 17 shows analogous fits for observed downflows. Near the surface $<I_{\text {down }}>$ decreases and $<V_{\text {down }}>$ increases, at $h \sim 120 \mathrm{~km}<I_{\text {down }}>$ increases and $<V_{\text {down }}>$ increases.

\subsection{Time evolution of velocity fields}

Up to now, we have studied the correlation between intensity and velocity fields acquired at the same time.
Now we examine the temporal evolution of velocity patterns calculated for the different photospheric lines. We tackle the problem of the dynamics of photospheric layers investigating:

1. the autocorrelation function $C(\tau)=\left\langle V_{\lambda}(t) V_{\lambda}(t+\tau)>\right.$ between velocity fields $V_{\lambda}$ obtained from the same line;

2. the cross-correlation function $C_{1,2}(\tau)=$ $<V_{\lambda 1}(t) V_{\lambda 2}(t+\tau)>$ between velocity fields $V_{\lambda 1}$ and $V_{\lambda 2}$ obtained from two combinations of lines, $\left[V_{538.0}, V_{537.9}\right]$ and $\left[V_{537.9}, V_{557.6}\right]$. The first combination characterizes the lower photosphere, while the second one describes the middle photosphere.

Using the time series of velocity fields relative to the same photospheric line, we studied the autocorrelation function $C(\tau)$ for the 12 successive velocity patterns (about $15 \mathrm{~min})$. For each line we obtained a total of $24 C(\tau)$ trends that are averaged and plotted in Fig. 18. In the case of the $\mathrm{C}$ I line the autocorrelation function has been fitted by a stretched exponential decay function whose general form is

$C(\tau)=\exp \left[-\left(\frac{\tau}{\tau_{0}}\right)^{\beta}\right]$,

with $\tau_{0}=[133 \pm 6] \mathrm{s}$ and $\beta=[0.46 \pm 0.02]$. Actually, the stretched exponential decay function, originally introduced by Kohlrausch in 1854 and rediscovered by Williams \& Watts in 1970, provides a good representation of experimental and numerical data in a wide variety of complex and out-of-equilibrium systems (Jund et al. 2001). For the Fe I data we use a simple exponential fit, obtaining as the characteristic time $\tau_{0}=[367 \pm 4 \mathrm{~s}] \sim 6 \mathrm{~min}$ for Fe I $537.9 \mathrm{~nm}$ line, and $\tau_{0}=[417 \pm 6 \mathrm{~s}] \sim 7 \mathrm{~min}$ for Fe I $557.6 \mathrm{~nm}$ line.

This different behaviour of the autocorrelation functions in low and mid photosphere suggests:

1. the occurrence of dynamical heterogeneity, i.e. a wide distribution of relaxation times, near the solar surface;

2 . the presence, in the middle photosphere, of a predominant characteristic time scale $(\sim 6-7 \mathrm{~min})$ for the evolution of the velocity fields; suggesting longer lifetimes for larger convective structures in the upper photosphere.

To evaluate the cross-correlation function $C_{1,2}(\tau)=$ $<V_{\lambda 1}(t) V_{\lambda 2}(t+\tau)>$, for each selected velocity frame $V_{\lambda 1}$, computed using the line $\lambda 1$ and at the time $t_{0}$, we used 9 different velocity maps, $V_{\lambda 2}$, as obtained from the line $\lambda 2$ at 9 different sampling time $\tau_{j}$, where $j \in$ $[-4,+4]$. Therefore, given $t_{0}$, we correlated upper velocity fields with $\tau_{j}$ ranging from previous $5 \mathrm{~min}$ to following $5 \mathrm{~min}$. The average of the $24 C_{1,2}(\tau)$ time crosscorrelation functions, relative to the selected combinations of lines, is shown in Fig. 19. On the basis of the two cross-correlation functions we were able to evaluate two different delay times, or phase differences, between the velocity fields in the two selected photospheric regions. 


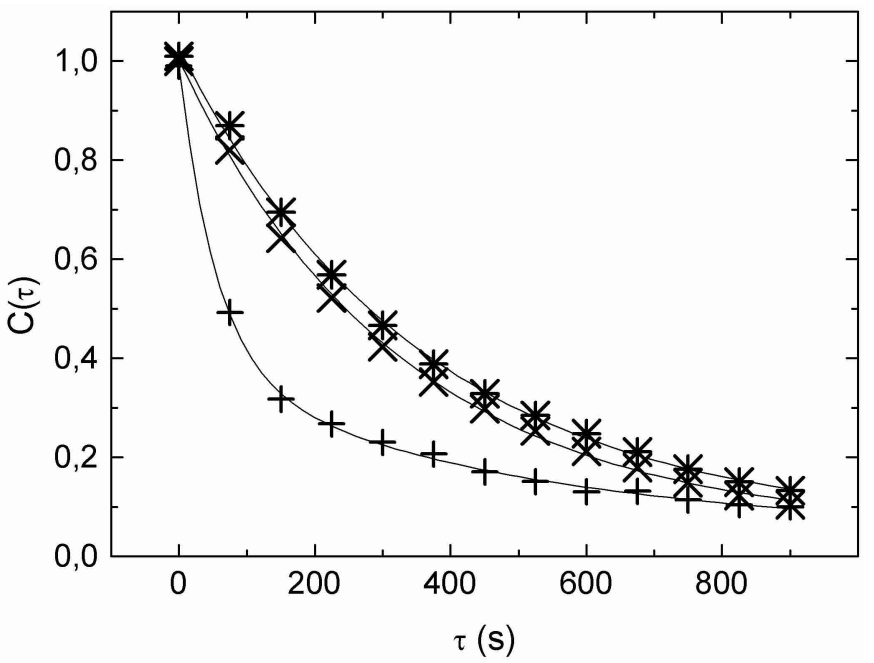

Fig. 18. $C(\tau)$ for the velocity maps obtained from the three observed lines. Symbols denote the measure of correlation for velocity fields at different sampling times; lines denote exponential fits of experimental data. To fit data derived from C I line observations we use a stretched exponential decay function, for Fe I data we use simple exponential functions. + crosses, C I $538.0 \mathrm{~nm}$ velocity map; × crosses, Fe I $537.9 \mathrm{~nm}$ velocity map; and stars, Fe I $557.6 \mathrm{~nm}$.

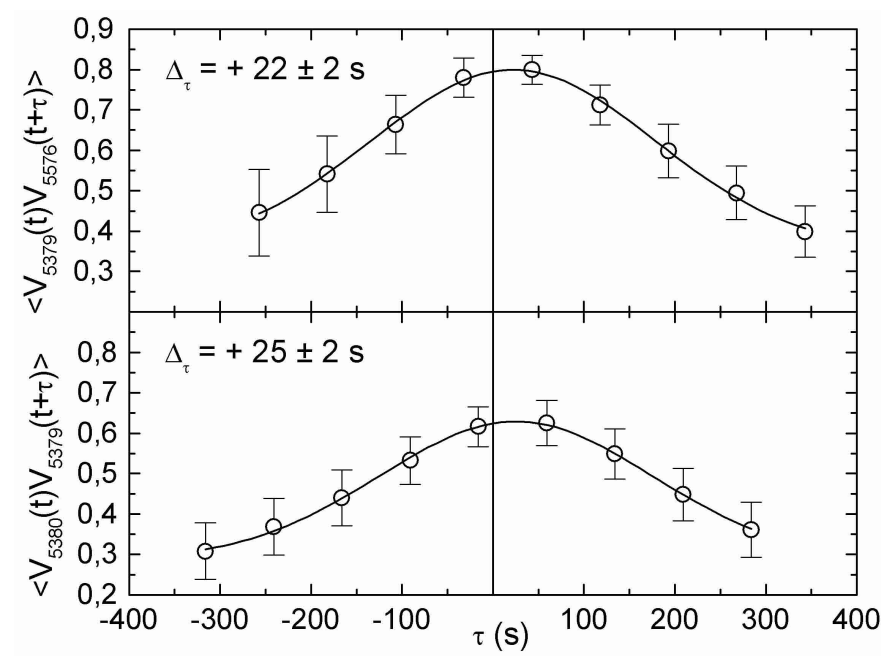

Fig. 19. The average of $24 C_{1,2}$ cross-correlation functions for the two photospheric layers. Points denote the measure of 4 previous, one simultaneous, and 4 following times, the associated error bars are fluctuations (rms). The sampling time is $75 \mathrm{~s}$. The solid lines indicate a Gaussian fit to determine the delay time.

For the $\left[V_{538.0}, V_{537.9}\right]$ combination we found a delay time $\Delta \tau=[25 \pm 2] \mathrm{s}$, while for the $\left[V_{537.9}, V_{557.6}\right]$ combination we found $\Delta \tau=[22 \pm 2] \mathrm{s}$. The delays suggest an upward propagation of the velocity fluctuation field.

\subsection{Time evolution of exploding granules}

Solar observations show a large variety of ways to vanish for the granular structures (e.g. merging, fading away, etc.). Among these, a very interesting one is the explosion

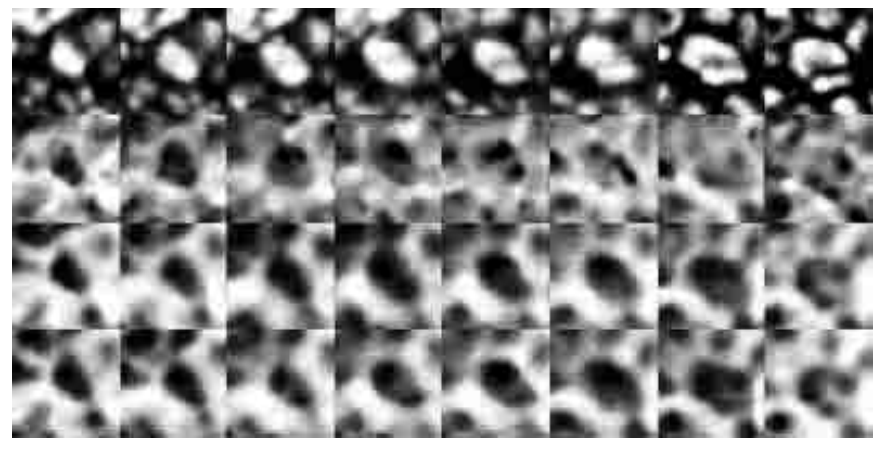

Fig. 20. Eight snapshots of the life of an undisturbed exploding granule. The time range is about $10 \mathrm{~min}$ and the size of the box is $6.8^{\prime \prime} \times 6.8^{\prime \prime}$. In the first row we show the sequence of broadband frames (solar surface). In the other rows we show the cospatial velocity frames derived from C I $538.0 \mathrm{~nm}$, Fe I $537.6 \mathrm{~nm}$ and Fe I $557.6 \mathrm{~nm}$ velocity fields. The velocity extent of the gray scale is from $-1000 \mathrm{~m} \mathrm{~s}^{-1}$ to $+1000 \mathrm{~m} \mathrm{~s}^{-1}$ for the $\mathrm{C}$ I line, and from $-500 \mathrm{~m} \mathrm{~s}^{-1}$ to $+500 \mathrm{~m} \mathrm{~s}^{-1}$ for Fe I lines. Notice the downflow ring-like features (bright regions in velocity frames) that break out around the upward fragments of exploder (dark regions in velocity frames).

of isolated granules into a set of small granular structures. To describe the structure and the dynamics of an exploding granule it is necessary not only to know its intensity history but also the velocity evolution in time and space. A qualitative description of an exploding granule, as observed in an intensity time series, can be summarized as follows. The granule increases its dimension and brightness very rapidly, develops a dark area in the center, and fragments in a set of small granules (typically 2 to 5 granules). Numerical simulations of exploding granule evolution (Arendt 1994; Rast 1995) suggest a decrease of the vertical velocity, in the central region, followed by plume formation. The experimental evidence for this fact has been obtained by Nesis (1994, private communication in Rast 1995), by using high-resolution spectrograms, and more recently, using spectroscopic imaging, by Berrilli et al. (2000), Hirzberger et al. (2001), and Roudier et al. (2001).

Here we focus our attention on the vertical structure and on the temporal evolution of two well-isolated and large exploding granules observed during our campaign. Using the coordinates of exploding granules, pointed out in broadband images, we extracted the corresponding velocity sub-fields. In Fig. 20 we report a full sequence of granule evolution as obtained from broadband images, and from the corresponding velocity sub-fields at different photospheric heights. For both exploders, as forerunner, it is possible to observe (Fig. 21) a deceleration in the center of the upward flow, even when the intensity profile does not present evidence of a central dark spot. When the inner dark spot appears, a downflow is observed in velocity maps. At the end of the fragmentation process a downflow ring $\left(\sim 4.7^{\prime \prime}\right)$ appears around the new granules. 


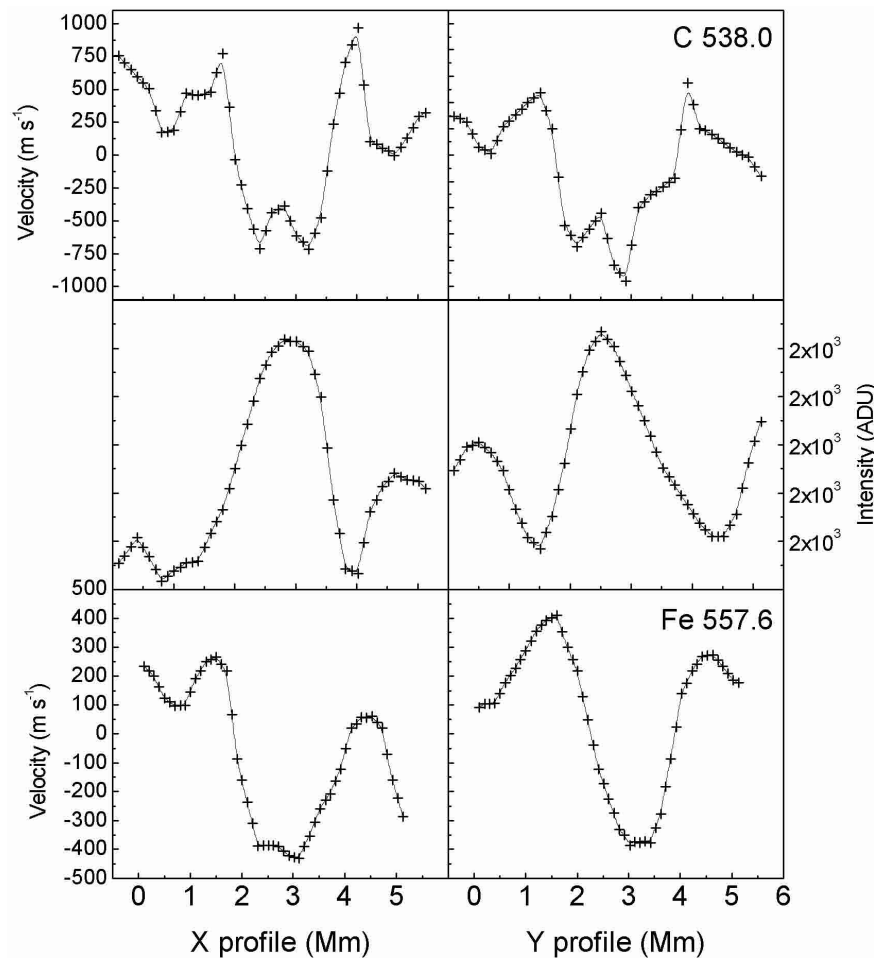

Fig. 21. Intensity and velocity profiles of the exploder reported in Fig. 20. The profiles cross the granule barycenter corresponding to the first column of Fig. 20. Upper panel: profiles of velocity field derived from C I line observations. Mid panel: profiles of broadband image. Lower panel: profiles of velocity field derived from Fe I line $(557.6 \mathrm{~nm})$ observations. Notice the presence of a central deceleration, in the different velocity profiles, even when the granule does not show evidence of a dark spot in the middle.

\section{Summary}

In this paper, we investigated different aspects of photospheric structures using multiline spectroscopy and broadband imaging. The photosphere was studied using velocity and centerline intensity frames derived from the observations of three absorption photospheric lines. The solar photospheric features were determined by means of automatic segmentation procedures applied to the photospheric frames (broadband intensity, velocity, centerline intensity). Our findings may be summarized as follows:

1. Both granules and cells, extracted from restored broadband images, show a steep drop in their area distributions, whose position corresponds to an effective size of $\sim 1 \mathrm{Mm}$, and of $\sim 1.5 \mathrm{Mm}$ for the granules and the larger cells, respectively. A qualitative agreement between the observed distribution of typical and/or big structures, and previously reported results (Title et al. 1989; Hirzberger et al. 1997) has been found.

2. The area distributions of the upflows and convective cells, as extracted from velocity patterns, show a shape similar to those relative to granules and granular cells. Moreover, in the case of velocity features, a dependence of upflows and convective cell area distributions on the photospheric height has been obtained. In the first layers of the solar atmosphere we found evidence of a selection effect, which removes small upward structures.

3. Numerical simulation of surface convection made by Gadun et al. (2000) are consistent with the observed relationship between average velocities and areas of identified upflows. Particularly, unstable models (fragmenting cells) produce averaged granular vertical velocities showing similar results to those obtained by observations of the C I $538.0 \mathrm{~nm}$ line.

4. Average velocities and centerline intensities for the selected velocity structures have been used to investigate the first layers of the photosphere. Using the larger velocity structures as a solar probe, we evaluated an asymptotic velocity by means of the relationship between the upward velocity $\left\langle V_{\text {up }}>\right.$ distributions for the three lines and the dimensions of the structures themselves. There is evidence of a very efficient upflow braking mechanism in the first photospheric layers. Particularly, we derived a deceleration of the mean asymptotic velocity corresponding to a variation of the mean upflow velocity of $\sim 300 \mathrm{~m} \mathrm{~s}^{-1}$ in the first $\sim 120 \mathrm{~km}$.

5. Using the velocity field autocorrelation functions and the cross-correlation functions between pair of velocity maps at different photospheric heights, typical characteristic times of photospheric patterns evolution have been inferred. The main result is that, near the solar surface, the dynamics resemble the behavior of a complex out-of-equilibrium system characterized by a dynamical heterogeneity. Conversely, in the mid atmosphere, where only one single characteristic time exists, the dynamical heterogeneity disappears. Moreover, the delay times evaluated from cross-correlation functions show that most of the dynamics develops in the lower layers of the photosphere.

6. We investigated both the temporal evolution and the vertical structure of two exploders using timeseries of broadband images and the related velocity fields in the different lines. A deceleration in the center of the upward flow before the appearance of dark areas in the broadband counterparts has been shown. This observation seems to confirm the evolutive framework reported in numerical simulations of exploding granule evolution (Rast 1995), suggesting a decrease of the vertical velocity in the central region followed by plume formation.

Acknowledgements. We thank the THEMIS staff for the efficient support in the observations and in particular we are grateful to Carine Briand, Guido Ceppatelli and Gianni Mainella. The fruitful comments of the unknown referee helped to improve the presentation of this work. We also thank Corrado Giammanco for the $k-\omega$ filter and Gino Smaldone for helpful suggestions. This work was partially supported by the Italian National Research Council (CNR) grant Agenzia2000 CNRC0084C4. 


\section{References}

Arendt, S. 1994, ApJ, 422, 862

Berrilli, F., Caccin, B., Cantarano, S., \& Egidi, A. 1997, Il Nuovo Cimento, 20, 297

Berrilli, F., Ermolli, I., Florio, A., \& Pietropaolo, E. 1999a, A\&A, 344, 965

Berrilli, F., Florio, A., Consolini, G., et al. 1999b, A\&A, 344, L29

Berrilli, F., Consolini, G., Florio, A., \& Pietropaolo, E. 2000, SAIt Meeting (M.Porzio, May 2000), Mem. of the Italian Astronomical Society, in press

Buonaura, B., \& Caccin, B. 1982, A\&A, 3, 113

Caccin, B., Gomez, J. N., Marmolino, C., \& Severino, G. 1977, A\&A, 54, 227

Canfield, R. C. 1976, Sol. Phys., 50, 239

Cavallini, F. 1998, A\&AS, 128, 589

Eibe, M. T., Mein, P., Roudier, Th., \& Faurobert, M. 2001, A\&A, 371, 1128

Faber, T. E. 1997, Fluid Dynamics for Physicist (Cambridge University Press)

Florio, A., \& Berrilli, F. 1998, Mem. SAIt 69, 655

Gadun, A. S., Solanki, S. K., \& Johannesson, A. 1999, A\&A, 350,1018

Gadun, A. S., Hanslmeier, A., Pikalov, K. N., et al. 2000, A\&AS, 146, 267

Gingerich, O., Noyes, R. W., Kalkofen, W., \& Cuny, Y. 1971, Sol. Phys., 18, 347

Gustafsson, B., \& Jorgensen, U. G. 1994, A\&AR, 6, 19

Hirzberger, J., Vazquez, M., Bonet, J. A., Hanslmeier, A., \& Sobotka, M. 1997, ApJ, 480, 406

Hirzberger, J., Koschinsky, M., Kneer, F., \& Ritter, C. 2001, A\&A, 367, 1011
Holweger, H., \& Müller, E. A. 1974, Sol. Phys., 39, 19

Hunt, B. R. 1984, Image Restoration in Digital Image Processing Techniques (Academic Press, Inc.)

Kim, Y.-C., Fox, P. A., Demarque, P., \& Sofia, S. 1996, ApJ, 461,499

Kippenhahn, R., \& Weigert, A. 1994, Stellar Structure and Evolution (Astronomy and Astrophysics Library, SpringerVerlag, Berlin Heidelberg New York)

Komm, R., Mattig, W., \& Nesis, A. 1989, A\&A, 221, 130

Krieg, J., Kneer, F., Koschinsky, N., \& Ritter, C. 2000, A\&A, 360,1157

Jund, P., Jullien, R., \& Campbell, I. 2001, Phys. Rev. E, 63, 036131

Nesis, A., \& Mattig, W. 1989, A\&A, 221, 130

Nesis, A., Hammer, R., Kiefer, M., et al. 1999, A\&A, 354, 265

Rast, P. 1995, ApJ, 443, 863

Rast, P. 1999, ApJ, 524, 462

Rimmele, T. R., Goode, P. R., Strous, L. H., \& Stebbins, R. T. 1995, ApJ, 444, 119

Roudier, Th., Eibe, M. T., Malherbe, J. M., et al. 2001, A\&A, 368,652

Shchukina, N. G., Bueno, J. T., \& Kostik, R. I. 1997, Sol. Phys., 172, 117

Spruit, H. C., Nordlund, Å., \& Title, A. M. 1990, ARA\&A, 28, 263

Steffen, M., Ludwig, H.-G., \& Kruss, A. 1989, A\&A, 213, 371

Stein, R. F., \& Nordlund, Å. 1998, ApJ, 499, 914

Strous, L. H., Goode, P. R., \& Rimmele, T. R. 2000, ApJ 200,58

Title, A. M., Tarbell, T. D., Topka, S. H., et al. 1989, ApJ, 336,475

Williams, G., \& Watts, D. C. 1970, Trans. Faraday Soc., 66, 80 